\title{
Non-Insulin Dependent Diabetes Mellitus and Alveolar Bone Loss Progression Over 2 Years
}

\author{
George W. Taylor, ${ }^{*}$ Brian A. Burt, ${ }^{\dagger}$ Mark P. Becker,${ }^{\dagger}$ Robert J. Genco, ${ }^{\ddagger}$ \\ Marc Shlossman, ${ }^{\S}$ William C. Knowler," and David J. Pettitt"
}

THIS STUDY TESTED THE HYPOTHESIS that persons with non-insulin dependent diabetes mellitus (NIDDM) have greater risk of more severe alveolar bone loss progression over a 2-year period than those without NIDDM. Data from the longitudinal study of the oral health of residents of the Gila River Indian Community were analyzed for 362 subjects, aged 15 to 57,338 of whom had less than $25 \%$ radiographic bone loss at baseline, and who did not develop NIDDM nor lose any teeth during the 2-year study period. The other 24 subjects had NIDDM at baseline, but met the other selection criteria. Bone scores (scale 0-4) from panoramic radiographs corresponded to bone loss of $0 \%, 1 \%-24 \%, 25 \%-49 \%, 50 \%-74 \%$, or $75 \%$ and greater. Change in bone score category was computed as the change in worst bone score (WBS) reading after 2 years. Age, calculus, NIDDM status, time to follow-up examination, and baseline WBS were explanatory variables in regression models for ordinal categorical response variables. NIDDM was positively associated with the probability of a change in bone score when the covariates were controlled. The cumulative odds ratio for NIDDM at each threshold of the ordered response was $4.23(95 \%$ C.I. $=1.80,9.92)$. In addition to being associated with the incidence of alveolar bone loss (as demonstrated in previous studies), these results suggest an NIDDM-associated increased rate of alveolar bone loss progression. J Periodontol 1998;69:76-83.

Key Words: Periodontitis; diabetes mellitus; diabetes mellitus, non-insulin dependent; longitudinal studies; epidemiology; alveolar bone loss.

Diabetes mellitus and adult periodontal disease are two relatively common chronic diseases in the United States population. Both conditions can produce disability and are presumed to be biologically linked. Though the precise mechanism is not known, the pathogenesis of periodontitis associated with diabetes mellitus is likely to be multifactoral. Several extensive reviews ${ }^{1-4}$ have mentioned the following mechanisms in explaining susceptibility of individuals with diabetes mellitus to periodontitis: 1) microangiopathy; 2) alterations in gingival crevicular fluid; 3) alterations in collagen metabolism; 4) altered host response; 5) altered subgingival microflora; and 6) hereditary patterns. Clinicians have long assumed that

\footnotetext{
*School of Dentistry, University of Michigan, Ann Arbor, MI. ${ }^{+}$School of Public Health.

‡State University of New York at Buffalo, NY.

sPreviously, State University of New York at Buffalo; currently, private practice, Chandler, AZ.

"National Institute of Diabetes and Digestive and Kidney Diseases, Phoenix, AZ.
}

individuals with diabetes mellitus have an increased risk for periodontitis, although the literature is actually much more equivocal, as discussed in reviews. ${ }^{4-6}$

Most literature about the diabetes mellitus-periodontitis relationship has addressed the association between periodontal diseases and insulin-dependent diabetes mellitus. Some of the studies of the insulin-dependent diabetes mellitus-periodontitis association report a positive relationship, while others report that no relationship exists. ${ }^{7-14}$ However, non-insulin dependent diabetes mellitus (NIDDM) occurs most frequently, ${ }^{15}$ but less has been written specifically about the NIDDM-periodontitis relationship. A review of reports that included subjects with diabetes and controls indentified few studies with the type(s) of diabetes specified. Three studies specified NIDDM: one in Yugoslavia, ${ }^{16}$ one in the Pima Indians of Arizona, USA, ${ }^{17-19}$ and one involving diabetic subjects in Minnesota, USA and Oulu, Finland. ${ }^{4,20}$ This set of studies collectively provides evidence that NIDDM, when compared to normal glucose tolerance, is associated with in- 
creased prevalence, severity, and incidence of periodontitis, although additional factors may be necessary for the NIDDM effect to become manifest.

Of the three studies cited with NIDDM subjects, only the study of the Pima Indians in Arizona has a longitudinal analysis estimating the effects of NIDDM on risk for periodontitis incidence. ${ }^{18}$ Nelson et al. provide ageand gender-adjusted incidence estimates of periodontitis, reporting that the incidence rate for subjects with NIDDM was $2.6(95 \%$ C.I. $=1.0,6.6)$ times that of those without NIDDM. This incidence rate ratio was derived from stratified contingency table analysis and calculated by using a modified Mantel-Hanzel statistic; hence, no longitudinal studies have modeled or quantified NIDDM-associated risk for severity of bone loss progression. The purpose of the present study was to extend these previous findings and to test the hypothesis that persons with NIDDM have greater risk of more severe alveolar bone loss progression than do those without NIDDM over a 2-year period. It also seeks to quantify that risk.

\section{MATERIALS AND METHODS}

Data for these analyses came from participants of the ongoing NIDDK longitudinal follow-up study of the Pima Indians in the Gila River Indian Community ${ }^{21}$ and the concurrent longitudinal oral health study conducted from 1982 to $1989 .{ }^{19}$ Subjects of the present study were of at least half Pima ancestry, aged 15 years or older, and examined approximately every 2 years. The examination included a medical history, physical examination, and review of outpatient and inpatient medical care records. Data were derived from 362 subjects, aged 15 to 57,338 of whom were free of diabetes at the beginning and did not develop NIDDM during a 2-year follow-up period. The other 24 subjects had NIDDM at baseline. Subjects selected were all those who had 20 or more teeth, lost no teeth during the study, and had less than $25 \%$ radiographic bone loss at baseline. Subjects who lost teeth during the follow-up period $(\mathrm{n}=37$ without NIDDM, $\mathrm{n}=5$ with NIDDM) were excluded because the degree of bone loss progression from baseline to the time of extraction and reasons for extraction of teeth were not available. Because there were few subjects with NIDDM in the study group who had no evidence of radiographic bone loss $(n=8)$, these inclusion criteria were established to obtain a cohort with minimal signs of bone loss at baseline. Subjects selected were those whose NIDDM status did not change over the period of observation in order to eliminate potential confounding associated with a change in exposure status. Diabetes was defined by a plasma glucose concentration $\geq 200 \mathrm{mg} / \mathrm{dl} 2$ hours after a 75 -g oral glucose load; subjects with normal glycemic control (NGT) and impaired glucose tolerance (IGT) had 2-hour plasma glucose concentrations $<140 \mathrm{mg} / \mathrm{dl}$ and 140 to $199 \mathrm{mg} / \mathrm{dl}$, respectively, and were classified as non-dia- betic. ${ }^{22}$ Venous blood was obtained 2 hours following ingestion of $75 \mathrm{~g}$ of carbohydrate. ${ }^{\square /}$ Plasma glucose was determined by the modified Hoffman method, using an automated analyzer."

All oral examinations were conducted by one of two trained and calibrated examiners who, at the time of examination, did not know the diabetes status of examined subjects. Clinical examination of periodontal status was performed on the six index teeth or their substitutes as described by Ramfjord. ${ }^{23}$ Calculus was scored using the calculus component of the periodontal disease index, ${ }^{23}$ plaque by the Silness-Löe plaque index,${ }^{24}$ and gingival inflammation by the Löe-Silness gingival index. ${ }^{25}$ Measures of probing depth were made on six sites per tooth, and loss of clinical attachment (LCA) on four sites per tooth.

Panoramic radiographs were used to assess interproximal bone loss for all teeth in the dentition, with measurements made using a modified Schei technique. ${ }^{19}$ To enhance consistency in radiograph exposures, all panoramic radiographs were made by a single technician on the same radiographic unit for the duration of the study. To standardize the radiographic procedure, all radiographs were exposed with the patient standing and stabilized with mounted hand grips; head alignment was accomplished using vertical and horizontal light beams in the radiographic unit to position the head with respect to anatomic landmarks; and a digital readout of the alignment was noted for subsequent exposures. All panoramic radiographs were evaluated and scored by the same examiner for the duration of the study. Intra-examiner reliability for the single examiner was assessed and determined to be acceptable (Cohen's Kappa $=0.88^{19}$ ). Both the technician and examiner were unaware of the diabetes status of the subjects.

The percentage of bone loss for each tooth was recorded on a $0-4$ ordinal scale, the score corresponding to percentage of radiographic bone loss. Bone scores (scale 0-4) from panoramic radiographs corresponded to bone loss of $0 \%, 1 \%-24 \%, 25 \%-49 \%, 50 \%-74 \%$, or $\geq 75 \%$. Change in bone score category was computed as the change in worst bone score (WBS) reading after 2 years. Change in WBS category, the outcome of interest in this analysis, is specified on a 4-category ordinal scale as no change; a 1-category increase; a 2-category increase; or a 3- or 4-category increase over baseline WBS.

Additional behavioral, medical, dental, and demographic variables were evaluated at their baseline values for confounding and effect modification in the analyses. These baseline covariates included self-reported alcohol consumption ( $\geq 3$ drinks per day); smoking (any smoking

IGlucola, Ames Co., Elkhart, IN.

"Dexcola, Custom Laboratories, Baltimore, MD

"Autoanalyzer, Technicon Instrument Corp., Tarrytown, NY. 
in the year preceding baseline); obesity (body mass index $>27 \mathrm{~kg} / \mathrm{m}^{2}$ ); systolic blood pressure (the first Korotkoff sound in the right arm with the subject in the supine position near the end of the medical examination); coronary artery disease, determined by presence of an abnormality on resting EKG according to the Whitehall criteria, ${ }^{26}$ age; and gender. Time to follow-up examination (in years) and number of teeth were also included as covariates.

Statistical analyses consisted of contingency table analysis and regression modeling. The contingency table analyses were used to examine data for sparseness and patterns suggesting possible confounding or effect modification. Regression modeling used an ordinal logistic regression model with cumulative logits ${ }^{27.28}$ to estimate the relative risk of change in WBS category associated with NIDDM and to calculate the probability of changes in WBS from the parameter estimates. It was not feasible to use change in maximum LCA as an outcome measure in these analyses because there was an insufficient number of subjects with baseline maximum LCA of $4 \mathrm{~mm}$ or less who also met the previously listed selection criteria. Age, number of teeth, systolic blood pressure, and time to follow-up examination were used in models as continuous covariates. Indicator variables were defined for alcohol consumption, gender, coronary artery disease, and smoking.

The regression models were developed in stages. First, using a forward stepwise selection procedure, PROC LOGISTIC, ${ }^{29}$ all variables considered to be important risk factors or confounders were included in a model with an indicator variable for NIDDM forced to remain in each model considered (the reference category included all non-diabetic subjects; i.e., those with either normal glucose tolerance or impaired glucose tolerance). This initial selection step resulted in a preliminary candidate model. Next, each of the variables that had been previously eliminated in the stepwise selection procedure was separately retested in this candidate model and retained if it attained a level of significance with $P$ value $<0.05$. Following testing for main effects, first order interaction terms were tested in the candidate model for relations between baseline age or diabetes and all the preceding main effects variables and for relations between baseline periodontal status and selected covariates. Quadratic terms were tested for all continuous covariates. Using likelihood ratio tests for the cumulative logistic regression models, terms found not to be statistically significant at the 0.05 level or important as effect modifiers or confounders in the modeled relationships were eliminated. Parameter estimate interpretation incorporated the point estimate as well as the $95 \%$ confidence interval in testing the ability to reject the null hypothesis. After obtaining a parsimonious model for this study's hypothesis, the model fit was evaluated using the likelihood ratio chi-squared test statistic,
Table 1. Baseline Descriptors Contrasting Subjects With and Without NIDDM (percentage of subjects in each row of each variable is shown in parentheses for non-diabetic and diabetic subjects)

\begin{tabular}{lcc}
\hline & \multicolumn{2}{c}{ Diabetes } \\
\cline { 2 - 3 } & Non-Diabetic & Diabetic \\
& $\mathrm{N}=338$ & $\mathrm{~N}=24$ \\
\hline Gender: male $\quad 138(40.8)$ & $8(33.3)$ \\
$\quad$ female & $200(59.2)$ & $16(66.7)$ \\
Age (median years) & 21.2 & 25.7 \\
Age categories & & \\
15-19 & $114(33.7)$ & $3(12.5)$ \\
$20-34$ & $194(57.4)$ & $17(70.8)$ \\
$35-57$ & $30(8.9)$ & $4(16.7)$ \\
Number of teeth (median) & 28 & 28 \\
Worst bone score & & \\
0\% bone loss & $189(55.9)$ & $8(33.3)$ \\
1-24\% bone loss & $149(44.1)$ & $16(66.7)$ \\
Calculus (median) & 0.28 & 0.36 \\
Gingival Index (median) & 1.61 & 1.61 \\
Plaque Index (median) & 1.17 & 1.28 \\
\hline
\end{tabular}

Table 2. Change in Worst Bone Score by Presence or Absence of Diabetes (percentage of subjects in each column are shown in parentheses for non-diabetic and diabetic subjects)

\begin{tabular}{|c|c|c|c|c|c|}
\hline \multirow[b]{2}{*}{ Diabetes } & \multicolumn{5}{|c|}{$\begin{array}{l}\text { Degree of Change in Worst Bone } \\
\text { Score at Follow-up: Number of } \\
\text { Categories of Change }\end{array}$} \\
\hline & None & 1 & 2 & 3 or 4 & $\mathrm{~N}$ \\
\hline Non-Diabetic & $201(59.5)$ & $126(37.3)$ & $7(2.1)$ & $4(1.2)$ & 338 \\
\hline Diabetic & $8(33.3)$ & $10(41.7)$ & $4(16.7)$ & $2(8.3)$ & 24 \\
\hline
\end{tabular}

score test for the proportional odds assumption, and residual analysis.

\section{RESULTS}

The strict selection criteria resulted in only 24 diabetic subjects being included. Table 1 , contrasting subjects with and without diabetes at baseline, shows more females than males were included although the proportions with NIDDM were similar. Diabetic subjects tended to be sightly older. There are notable differences in WBS; a higher proportion of subjects with NIDDM had $1 \%$ to $24 \%$ radiographic bone loss (67\%) than did non-diabetics (44\%). Table 1 also shows slight differences in calculus and plaque indices between subjects with and without diabetes and no difference for median number of teeth and gingival index. Table 2 shows the crude relationship between diabetes and degree of change in WBS; overall, a higher proportion of subjects with NIDDM were in each category experiencing change in WBS, and a smaller proportion of subjects with NIDDM had no change in WBS (33\% vs. 59\%). Table 3 shows the frequency distribution of WBS category changes after 2 years follow-up, stratified by age. Though many of the cells have sparse data, there are higher proportions of diabetic subjects with bone loss in the two youngest age strata, while within the oldest age stratum, a smaller proportion of subjects with 
Table 3. Change in Worst Bone Score, by Presence or Absence of Diabetes and Baseline Age (percentage of subjects in each column are shown in parentheses for non-diabetic and diabetic subjects)

\begin{tabular}{|c|c|c|c|c|c|c|}
\hline \multirow[b]{2}{*}{ Diabetes } & \multirow{2}{*}{$\begin{array}{l}\text { Baseline } \\
\text { Age }\end{array}$} & \multicolumn{5}{|c|}{$\begin{array}{c}\text { Degree of Change in Worst Bone Score at Follow-up: } \\
\text { Number of Categories of Change }\end{array}$} \\
\hline & & None & 1 & 2 & 3 or 4 & $\mathrm{~N}$ \\
\hline \multirow[t]{3}{*}{ Non-Diabetic } & $15-19$ & $82(71.9)$ & $30(26.3)$ & $2(1.8)$ & 0 & 114 \\
\hline & $20-34$ & $110(56.7)$ & $78(40.2)$ & $4(2.1)$ & $2(1.0)$ & 194 \\
\hline & $35-57$ & $9(30.0)$ & $18(60.0)$ & $1(3.3)$ & $2(6.7)$ & 30 \\
\hline \multirow[t]{3}{*}{ Diabetic } & $15-19$ & $1(3.3)$ & $1(33.3)$ & 0 & $1(33.3)$ & 3 \\
\hline & $20-34$ & $5(29.4)$ & $9(52.9)$ & $2(11.8)$ & $1(5.9)$ & 17 \\
\hline & $35-57$ & $2(50.0)$ & 0 & $2(50.0)$ & 0 & 4 \\
\hline
\end{tabular}

Table 4. Change in Worst Bone Score, by Diabetes Status and Baseline Worst Bone Score (percentage of subjects in each column are shown in parentheses for non-diabetic and diabetic subjects)

\begin{tabular}{llrrrrr}
\hline & & \multicolumn{5}{c}{ Degree of Change in Worst Bone Score at Follow-up: } \\
& Baseline & \multicolumn{5}{c}{ Number of Categories of Change } \\
\cline { 2 - 7 } Diabetes & WBS & \multicolumn{1}{c}{ None } & 1 & 2 & 3 or 4 & N \\
\hline Non-Diabetic & $0 \%$ & $100(52.9)$ & $80(42.3)$ & $7(3.7)$ & $2(1.1)$ & 189 \\
& $1-24 \%$ & $101(67.8)$ & $46(30.9)$ & 0 & $2(1.3)$ & 149 \\
Diabetic & $0 \%$ & $2(25.0)$ & $4(50.0)$ & $1(12.5)$ & $1(12.5)$ & 8 \\
& $1-24 \%$ & $6(37.5)$ & $6(37.5)$ & $3(18.7)$ & $1(6.2)$ & 16 \\
\hline
\end{tabular}

Table 5. Summary Table of Change in Worst Bone Score for Cumulative Regression Models $(\mathbf{N}=\mathbf{3 6 2})$

\begin{tabular}{|c|c|c|c|c|c|c|}
\hline & \multicolumn{3}{|c|}{ Main Effects Model* } & \multicolumn{3}{|c|}{ Interaction Model $\dagger$} \\
\hline & $\begin{array}{l}\text { Coefficient } \\
\text { Estimate }\end{array}$ & $P$-value $\ddagger$ & $\begin{array}{l}\text { Odds Ratio } \\
\text { (95\% C.I.) }\end{array}$ & $\begin{array}{c}\text { Coefficient } \\
\text { Estimate }\end{array}$ & $P$-value + & $\begin{array}{l}\text { Odds Ratio } \\
\text { (95\% C.I.) }\end{array}$ \\
\hline \multicolumn{7}{|l|}{ Main Effects } \\
\hline Intercept 1 & -3.216 & .0001 & & -3.601 & .0001 & \\
\hline Intercept 2 & -6.347 & .0001 & & -6.780 & .0001 & \\
\hline Intercept 3 & -7.476 & .0001 & & -7.904 & .0001 & \\
\hline NIDDM & 1.442 & .0009 & $4.23(1.80,9.92)$ & 5.817 & .0001 & see Table 6 \\
\hline Age (years) & 0.095 & .0001 & $1.09(1.06,1.14)$ & .1122 & .0001 & see Table 6 \\
\hline Time to follow-up & 0.305 & .0033 & $1.36(1.11,1.66)$ & .3156 & .0027 & $1.37(1.12,1.68)$ \\
\hline Worst bone score & -1.611 & .0001 & $0.20(0.11,0.35)$ & -1.680 & .0001 & $0.19(0.11,0.33)$ \\
\hline Calculus & 1.045 & .0002 & $2.84(1.65,4.88)$ & 1.010 & .0003 & $2.74(1.59,4.73)$ \\
\hline \multicolumn{7}{|l|}{ Interaction } \\
\hline NIDDM•AGE & & & & -.1561 & .0017 & see Table 6 \\
\hline
\end{tabular}

*Model evaluation statistics: likelihood ratio Chi-squared statistic $=73.595(5 \mathrm{df}), P=.0001$; score test for the proportional odds assumption $=$ $15.097, P=.1286$

$\dagger$ Model evaluation statistics: likelihood ratio Chi-squared statistic $=82.289(6 \mathrm{df}), P=.0001$; score test for the proportional odds assumption $=$ $24.290, P=.0174$.

$\ddagger P$-value of the Wald Chi-squared statistic with respect to a Chi-squared distribution with one degree of freedom.

NIDDM shows a change in WBS category. For subjects who experience bone loss, there is a tendency for the bone loss to be more severe among those with NIDDM as evidenced by higher proportions of subjects with NIDDM in each category of bone score change. Table 4 shows the frequency distribution of WBS category changes stratified by diabetes and baseline WBS. Although the number of subjects is sparse in several cells, this table shows that a greater proportion of subjects, regardless of glucose tolerance status, had bone loss progression when the baseline WBS was $0 \%$ rather than $1 \%$ to $24 \%$.

Table 5 summarizes the results of the final cumulative logistic regression models used to model NIDDM-asso- ciated risk for alveolar bone loss. The coefficient estimates, model evaluation statistics, odds ratios, and $P$ values obtained from using a main effects model and a model including a term for the interaction between NIDDM and baseline age are shown. Both models show that NIDDM is a significant risk factor for alveolar bone loss, as well as for more severe bone loss progression at follow-up when controlled for the other covariates. Additional covariates that were significantly associated with alveolar bone loss progression included time to followup, and baseline values for age, calculus, and WBS. Covariates found not to be significant in the models were alcohol consumption, smoking, obesity, systolic blood 


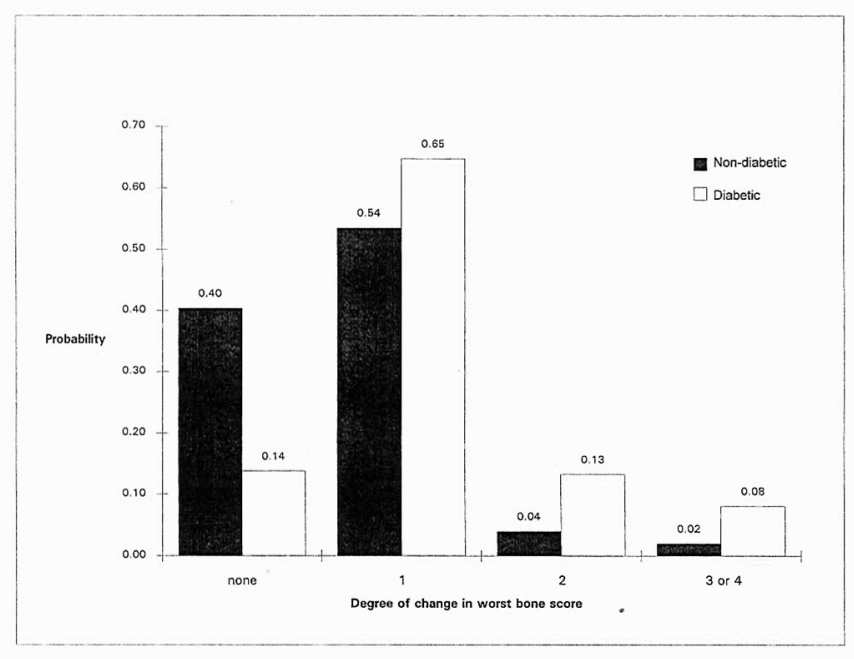

Figure 1. Probability of change in worst bone score.

Table 6. Age-Specific Odds Ratios and 95\% Confidence Limits for NIDDM-Associated Change in Worst Bone Score Category

\begin{tabular}{lccc}
\hline \multirow{2}{*}{$\begin{array}{l}\text { Baseline } \\
\text { Age }\end{array}$} & Odds Ratio & Lower Limit & Upper Limit \\
\cline { 3 - 4 } & 32.31 & 7.22 & 144.57 \\
15 & 6.78 & 2.76 & 16.70 \\
25 & 1.42 & 0.46 & 4.40 \\
35 & 0.30 & 0.04 & 2.01 \\
45 & 0.06 & 0.00 & 1.04 \\
55 & & \\
\hline
\end{tabular}

pressure, coronary artery disease, gender, and number of teeth. Figure 1 contrasts the probabilities of change in bone score category estimated from the main effects cumulative logistic regression model for subjects with and without diabetes. It shows that the probability of a 1-, 2-, or 3-, or 4-category change in radiographic worst bone score was greater for subjects with NIDDM (e.g., subjects with NIDDM had a probability of 0.13 for a 2-category change, while the probability for those without diabetes was 0.04$)$. The interaction model suggests that the effect of NIDDM on alveolar bone loss progression may be lower or non-existent at older ages. The age-specific odds ratios for alveolar bone loss progression estimated from the interaction model are presented in Table 6. These data show that the risks for alveolar bone loss progression decrease in the older categories. The wide confidence intervals resulted from the sparse data as seen in Table 3.

\section{DISCUSSION}

The results from this study support the hypothesis that NIDDM is a significant risk factor for more severe alveolar bone loss progression within approximately 2 years of follow-up. This result extends previous findings of NIDDM as a risk indicator of worse periodontal health as shown in cross-sectional studies ${ }^{4.16 .17,19,20}$ and as a risk factor for increased incidence of periodontal disease. ${ }^{18}$

For hypothesis testing in these analyses, the cumulative logistic regression model was used. This model is particularly useful when there is reason to believe that the response of interest has been derived from categorizing an underlying continuous response, as is the case in constructing the ordinal categories for degree of change in radiographic worst bone score at follow-up. Use of cumulative logits in this analysis provided a way to analyze change of periodontal status over time, where change in periodontal status in subjects was ordinally defined at follow-up as no change, or a 1-, 2-, 3- or 4-category increase. Parameter estimates obtained from this approach also allowed estimating the probability of making a 1category leap, 2-category leap, and so on, over the 2-year follow-up period (Fig. 1).

These analyses have used both the main effects and the interaction models to assess risk for alveolar bone loss progression. Evaluated together, they provide complementary information in understanding the relationship between NIDDM and progression of periodontal bone loss. Both models fit the data well and have stable parameter estimates for the main effects covariates. The main effects model suggests that the odds of all categories of change are constant and greater in subjects with NIDDM (odds ratio $=4.23 ; 95 \%$ C.I. $1.80-9.92$ ). This model estimated higher probabilities for each category of radiographic bone score change in subjects with NIDDM (Fig. 1). The interaction model (Table 5) suggests that the effects of NIDDM on change in WBS may be lower or non-existent in the higher ages. This finding is similar to that of Thorstensson and Hugoson, ${ }^{30}$ who found that while younger subjects with diabetes had significantly more bone loss than controls, older subjects did not. Table 6 shows the change in odds ratio for NIDDM's effect as age increases. This is derived from the significant NIDDM-age interaction term estimated in the model. Because both models provide insight into the incidence as well as progression of periodontal bone loss, both were retained. Finding an NIDDM-age interaction may have, to some extent, reflected a selection bias resulting from the strict selection criteria; i.e., older subjects with NIDDM who met the criteria for selection (acceptable baseline WBS, 20 or more teeth, and not losing teeth during the follow-up period) may have been individuals who were less susceptible to periodontitis and its progression. These selection criteria eliminated the pool of older subjects who had already experienced more loss of alveolar bone than was acceptable for inclusion in the analyses or experienced tooth loss during the follow-up period, thus resulting in a smaller or apparently protective effect estimated for NIDDM in the remaining older subjects selected. An alternative or supplemental explanation takes into account the more common occurrence of periodontitis in older ages than at younger ages, and its more common occurrence in diabetics than non-diabetics. At younger ages periodontitis is less common in non-diabetic subjects, 
thereby leading to a large difference between diabetics and non-diabetics-ergo a "large diabetes effect." On the other hand, at older ages the non-diabetic subjects are more likely to have periodontitis; therefore, the difference between non-diabetics and diabetics will be less, resulting in a "smaller diabetes effect." Nevertheless, the NIDDM-age interaction should be interpreted cautiously because the data generating the NIDDM-age interaction are sparse in several cells (Table 3), and could have produced a spurious relationship between NIDDM and baseline age.

Results from our cumulative logistic models also suggest that there may be an inverse relationship between baseline WBS and change in WBS at follow-up; i.e., subjects with no measurable radiographic bone loss at baseline (maximum WBS $=0 \%$ ) have a higher risk of change in WBS than do subjects with maximum WBS of $1 \%$ to $24 \%$ at baseline. This finding is consistent with Papapanou and Wennstrom, ${ }^{31}$ who also found an inverse relationship between baseline radiographic bone level and amount of bone loss at follow-up. On the other hand, Albandar ${ }^{32}$ reported a direct relationship between degree of bone loss at baseline and at follow-up. These seemingly divergent findings may be related to the unit of analysis. In the present study, as was the case with Papapanou and Wennstrom, ${ }^{31}$ the individual is the analytic unit rather than the tooth or site, as was the case in Albandar. ${ }^{32} \mathrm{Al}-$ though a biological interpretation is speculative, it is possible that the finding in the present study reflects an increased risk of change in bone score at the individual level in subjects who have minimal radiographic evidence of bone loss at baseline because these individuals have more teeth at risk to contribute to a change in WBS. In contrast, subjects already having had bone loss at baseline may have fewer teeth susceptible to further disease episodes within the follow-up period, thus tending to lessen the likelihood of a further change in WBS during the period of observation.

In this study of the effects of NIDDM on incidence and progression of alveolar bone loss, several potential sources of bias and their effects have been considered. First, subject selection was stringent to reduce extraneous effects of outside variables as far as possible. The tradeoff was a considerable reduction in the $N$ for analysis and a potentially weakened ability to detect real differences between the groups. As previously described, those selected for this analysis were subjects with 20 or more teeth at baseline, who lost no teeth during the follow-up period, had baseline WBS of $24 \%$ or less, and whose diabetes status did not change during the follow-up period. These selection criteria resulted in only a small number of subjects with NIDDM being included, and the proportion of NIDDM subjects in these analyses is much smaller than the overall proportion of NIDDM subjects in this community. Consequently, the possibility of selec- tion bias is recognized. While such a bias is indeed likely, it is toward selecting the "healthiest" of the NIDDM subjects, and hence against rejecting the null hypothesis. Since the results permitted rejecting the null hypothesis, the potential selection bias has actually provided more confidence in the conclusion than had the selection criteria been broadened to include more "dentally compromised" NIDDM subjects in this analysis.

Prior to accepting the results from the models presented, several tests were performed to assess the effects of the stringent selection criteria. Separate data sets with larger $N$ 's were created to test the consistency of the NIDDM effect where baseline WBS was $\leq 2(0 \%-49 \%)$ instead of $\leq 1(0 \%-24 \%)$; where subjects with tooth loss were not excluded; and where subjects with a change in glucose tolerance status were included. The results (not shown) of models incorporating these relaxed inclusion criteria were not substantially different from those presented here. Hence, the results of the more conservative analyses have been reported. Particular emphasis was placed on handling tooth loss because previous longitudinal studies of radiographic bone loss or attachment loss did not account for the effects of losing teeth on change in periodontal status, thus potentially biasing any measurements of loss (or gain) in an unknown manner. Two of the prior Pima Indian reports included tooth loss as a measure of periodontitis by assigning all missing teeth the maximum radiographic worst bone score. ${ }^{18,19}$ This classification is one which is subject, in a substantial way, to the judgment of clinicians and the dentist-patient interactions. Given the severity of periodontitis which is commonly seen in this population, this classification was probably correct in many cases, but it could also have led to substantial misclassification and potential bias. The approach in the present study was therefore to exclude this common clinical treatment for periodontitis (i.e., tooth extraction) from the objective measure of the conditionradiographic bone loss.

Another potential source of bias in testing the hypothesis was created by combining the NGT and IGT categories to contrast the effects of NIDDM and nonNIDDM, the concern being that individuals with IGT have greater risk for developing NIDDM. Thus, in combining the NGT and IGT groups, any effects of NIDDM may have been attenuated or obscured in the statistical models. To test whether IGT had an effect different from NGT, the final models obtained from the hypothesis testing procedures using the dichotomous NIDDM/nonNIDDM variable were re-tested using separate dummy variables for IGT and NIDDM. The results (not shown) indicated no significant differences between the effects of NGT and IGT, thus supporting the rationale for combining these two glucose tolerance categories in the regression models presented.

A related issue is the role of glycemic control in the 
risk for occurrence and progression of periodontitis in individuals with NIDDM. We conducted a separate analysis to disaggregate subjects with NIDDM into those with better control (glycosylated hemoglobin A1 <9\%) and poorer control (glycosylated hemoglobin $\mathrm{A} 1 \geq 9 \%$ ). The final cumulative logistic regression model in that analysis also included age, calculus, time to follow-up examination, and baseline WBS as statistically significant explanatory variables. Poorer control was positively associated with greater risk for a change in bone score compared to subjects without NIDDM; the cumulative odds ratio at each threshold of the ordered response was 11.4 (95\% C.I. $=$ 2.5, 53.3). The cumulative odds ratio for subjects with better control was $2.2(95 \%$ C.I. $=0.7,6.5)$, when contrasted with those without NIDDM. These results suggest there may be a gradient for the risk of bone loss progression related to degree of glycemic control; the risk for subjects with better. control, although less than for those with poorer control, may still be greater than for those without NIDDM..$^{33}$

Data used for assessment of the outcome (change in radiographic bone score) probably incorporated some of the types of biases which are inherent in the use of radiographs for measuring bone loss. Several studies over the past three decades, using different approaches, have evaluated the accuracy of panoramic radiography in determining periodontal bone destruction. ${ }^{34-41}$ Collectively, these reports provide substantial evidence to support use of panoramic radiographs for epidemiologic studies of periodontal bone loss. While some degree of measurement error is inevitable in this study, the misclassifications resulting from error in measurements of bone loss using the panoramic radiographs would be non-differential; i.e., randomly distributed among those subjects with and without NIDDM. This type of bias; i.e., non-differential misclassification, would tend to bias associations towards the null, leading to a finding of no difference in progression of bone loss between those with and without NIDDM if the measurement error was extensive.

While this detailed analysis leads to confidence in the internal validity of the conclusions reached, a major consideration is the generalizibility of results derived from studying the Gila River Indian Community population. An important question is whether the diseases under study have the same pathogenesis and natural history in this community as they do elsewhere, or whether there is something unique about these diseases in the Pima Indians. The complications of diabetes were found to be similar in the Gila River Indian Community as in other populations, ${ }^{42}$ though NIDDM occurs at a younger age in Pima Indians than in U.S. whites, and diabetic nephropathy has a greater impact on mortality than cardiovascular disease in Pima Indians with NIDDM compared with U.S. whites with NIDDM. ${ }^{43}$ With periodontitis, there were marked differences in the predominant cultivable micro- flora between diabetic and non-diabetic periodontitis patients in the Gila River community, but the diabetes-related serogroup of Porphyromonas gingivalis found among the Pima was not unique to them. ${ }^{44}$ Emrich et al. ${ }^{17}$ observed that the clinical presentation of adult periodontitis in the Pima Indians did not differ from that in other populations.

The combined medical database and oral health dataset from the Gila River project used for this study have provided a study sample from a well-defined population to assess the association of periodontitis with NIDDM longitudinally. The modeling procedures applied in this study have allowed testing a hypothesis not previously tested. This study's findings have major implications for clinical management protocols of the diseases. Dentists and physicians treating patients with NIDDM should be aware that periodontitis is another non-specific complication in their patients, and dentists should be aware that patients with NIDDM are at increased risk for more severe progression of periodontitis.

It is concluded that in addition to being associated with increased incidence of bone loss, NIDDM also increases the risk for more severe progression of alveolar bone loss. The effect of NIDDM on progression of alveolar bone loss may not be constant at all ages.

\section{Acknowledgments}

This study was supported by the Michigan Diabetes Research and Training Center and by NIDR grants DE06514 and DE07157. We wish to acknowledge Dr. Lynn Budding for his consultation and field work in helping to collect the oral health data; Ms. Ana Cabrera-Arevalo for assistance in data management and computer programming; Dr. Peter H. Bennett for his advice and consultation; and the members and leaders of the Gila River Indian Community for generously participating in and encouraging the study.

\section{REFERENCES}

1. Wilton JMA, Griffiths GS, Curtis MA, et al. Detection of high-risk groups and individuals for periodontal diseases. $J$ Clin Periodontol 1988;15:339-346.

2. Murrah VA. Diabetes mellitus and associated oral manifestations: A review. J Oral Pathol 1985;14:271-281.

3. Manouchehr-Pour M, Bissada NF. Periodontal disease in juvenile and adult diabetic patients: A review of the literature. $J$ Am Dent Assoc 1983;107:766-770.

4. Oliver RC, Tervonen T. Diabetes-A risk factor for periodontitis in adults? J Periodontol 1994;65:530-538.

5. Szpunar SM, Ismail AI, Eklund SA. Diabetes and periodontal disease: Analyses of NHANES I and HHANES. J Dent Res 1989; 68(Spec. Issue):383(Abstr. 1605).

6. Katz PP, Wirthlin MR, Szpunar SM, Selby JV, Sepe S, Showstack JA. Periodontal disease in persons with diabetes: Epidemiology and prevention. Diabetes Care 1991;14:375-385.

7. Cianciola LA, Park BH, Bruck E, Mosovich L, Genco RJ. Prevalence of periodontal disease in insulin-dependent diabetes mellitus (juvenile diabetes). J Am Dent Assoc 1982;104:653-660. 
8. Goteiner D, Vogel R, Deasy M, Goteiner C. Periodontal and caries experience in children with insulin-dependent diabetes mellitus. $J$ Am Dent Assoc 1986;113:277-279.

9. Harrison R, Bowen WH. Periodontal health, dental caries, and metabolic control in insulin-dependent diabetic children and adolescents. Pediatr Dent 1987;9:283-286.

10. Kjellman O, Henriksson CO, Bergnagen N, Andersson B. Oral conditions in 105 subjects with insulin-treated diabetes mellitus. Swed Dent J 1970;63:99-110.

11. Novaes AB Jr., Pereira AL, de Moraes N, Novaes AB. Manifestations of insulin-dependent diabetes mellitus in the periodontium of young Brazilian patients. J Periodontol 1991;62:116-122.

12. Rylander H, Ramberg P, Blohme G, Lindhe J. Prevalence of periodontal disease in young diabetics. J Clin Periodontol 1987;14:3843.

13. Sandholm L, Swanljung O, Rytomaa I, Kaprio EA, Maenpaa J. Periodontal status of Finnish adolescents with insulin-dependent diabetes mellitus. J Clin Periodontol 1989;16:617-620.

14. De Pommereau V, Dargent-Pare C, Robert JJ, Brion M. Periodontal status in insulin-dependent diabetic adolescents. $J$ Clin Periodontol 1992;19:628-632.

15. Harris MI. Prevalence of noninsulin-dependent diabetes and impaired glucose tolerance. In: Harris MI, ed. Diabetes in America. NIH Publ. No. 85-1468. Washington, DC: Government Printing Office; 1985:VI-1-31

16. Bacic M, Plancak D, Granic M. CPITN assessment of periodontal disease in diabetic patients. J Periodontol 1988;59:816-822.

17. Emrich LJ, Shlossman M, Genco RJ. Periodontal disease in noninsulin-dependent diabetes mellitus. J Periodontol 1991;62:123130.

18. Nelson RG, Shlossman M, Budding LM, et al. Periodontal disease and NIDDM in Pima Indians. Diabetes Care 1990;13:836-840.

19. Shlossman M, Knowler WC, Pettitt DJ, Genco RJ. Type 2 diabetes mellitus and periodontal disease. J Am Dent Assoc 1990;121:532536.

20. Oliver RC, Tervonen T. Periodontitis and tooth loss: Comparing diabetics with the general population. J Am Dent Assoc 1993;124:7176.

21. Knowler WC, Bennett PH, Hamman RF, Miller M. Diabetes incidence and prevalence in Pima Indians: A 19-fold greater incidence than in Rochester, Minnesota. Am J Epidemiol 1978;108:497-505.

22. World Health Organization. WHO Expert Committee on Diabetes Mellitus. Second Report. Technical Report Series 646:1-80. Geneva: World Health Organization; 1980.

23. Ramfjord SP. Indices for prevalence and incidence of periodontal disease. J Periodontol 1959;30:51-59.

24. Silness J, Löe H. Periodontal disease in pregnancy. II. Correlation between oral hygiene and periodontal condition. Acta Odontol Scand 1964;22:112-135.

25. Löe H, Silness, J. Periodontal disease in pregnancy. I. Prevalence and severity. Acta Odontol Scand 1963;21:533-551.

26. Fuller JH, McCartney P, Jarrett RJ, et al. Hyperglycaemia and coronary heart disease: The Whitehall study. J Chron Dis 1979;32:721728.

27. Agresti A. Categorical Data Analysis. New York: John Wiley and Sons; $1990: 322-326$
28. Koch GC, Beck JD. Statistical methodologies useful for the analysis of data from risk-assessment studies. J Public Health Dent 1992;3: $146-167$

29. SAS Institute Inc. SAS Technical Report P-200, SAS/STAT Software: Calis and Logistic Procedures, Release 6.04. Cary, NC: SAS Institute Inc.; 1990:175-230.

30. Thorstensson $\mathbf{H}$, Hugoson A. Periodontal disease experience in adult long-duration insulin-dependent diabetics. J Clin Periodontol 1993; 20:352-358

31. Papapanou PN, Wennstrom JL. A 10-year retrospective study of periodontal disease progression. Clinical characteristics of subjects with pronounced and minimal disease development. $J$ Clin Periodontol 1990;17:78-84.

32. Albandar JM. Some predictors of radiographic alveolar bone height reduction over 6 years. J Periodont Res 1990;25:186-192.

33. Taylor GW, Burt BA, Becker MP, Genco RJ, Shlossman M. Glycemic control and alveolar bone loss progression in Type II diabetes. Ann Periodontol Accepted for publication.

34. Ainamo J, Tammisalo EH. The orthopantomogram in quantitative assessment of marginal bone loss. Suom Hammaslaak Toim 1967; 63:132-138.

35. Kaimenyi JT, Ashley FP. Assessment of bone loss in periodontitis from panoramic radiographs. J Clin Periodontol 1988;15:170-174.

36. Akesson L, Hakansson J, Rohlin M. Comparison of panoramic and intraoral radiography and pocket probing for the measurement of the marginal bone level. J Clin Periodontol 1992;19:326-332.

37. Rohlin M, Akesson L, Hakansson J, Hakansson H, Nasstrom K. Comparison between panoramic and periapical radiography in the diagnosis of periodontal bone loss. Dentomaxillofac Radiol 1989; 18:72-76.

38. Akesson L, Rohlin M, Hakansson J. Marginal bone in periodontal disease: An evaluation of image quality in panoramic and intra-oral radiography. Dentomaxillofac Radiol 1989;18:105-112.

39. Bjorn A, Holmberg K. Radiographic determination of periodontal bone destruction in epidemiological research. Odontologisk Revy 1966; 17:232-250.

40. Bjorn AL, Bjorn $\mathrm{H}$, Halling A. An abbreviated index for periodontal bone height. Odontol Revy 1975;26:225-230.

41. Ahlqwist M, Halling A, Hollender L. Rotational panoramic radiography in epidemiological studies of dental health. Comparison between panoramic radiographs and intraoral full mouth surveys. Swed Dent $J$ 1986;10:73-84.

42. Knowler WC, Pettitt DJ, Saad MF, Bennett PH. Diabetes mellitus in the Pima Indians: Incidence, risk factors and pathogenesis. Diabetes Metab Rev 1990;6:1-27.

43. Sievers ML, Nelson RG, Knowler WC, Bennett PH. Impact of NIDDM on mortality and causes of death in Pima Indians. Diabetes Care 1992;15:1541-1549.

44. Zambon JJ, Reynolds H, Fisher JG, Shlossman M, Dunford R, Genco RJ. Microbiological and immunological studies of adult periodontitis in patients with noninsulin-dependent diabetes mellitus. $J$ Periodontol 1988;59:23-31.

Send reprint requests to: Dr. George W. Taylor, Department of Cariology, Restorative Sciences, and Endodontics, University of Michigan School of Dentistry, 1011 N. University, Ann Arbor, MI 48109. Accepted for publication May 22, 1997. 